\title{
Gauge Boson Production at the Tevatron
}

\section{Daniel Elvira* $\dagger$}

Fermi National Accelerator Laboratory, P.O.Box 500, Batavia, IL 60510, USA

\section{E-mail: danielofnal.govi}

ABSTRACT: We present new measurements on gauge boson production from data taken during 1994-1996 by the $\mathrm{D} \varnothing$ detector: the differential production cross section of the $W$

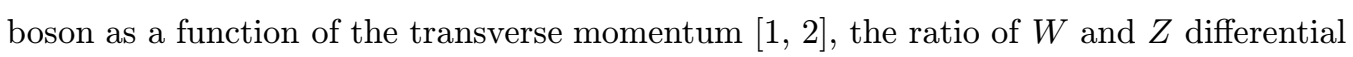
cross sections $\left[\begin{array}{l}3 \bar{v} \\ \mathfrak{v}\end{array}\right.$ cross section at a $p \bar{p}$ center-of-mass energy of $630 \mathrm{GeV}$, and the ratio of photon crosssections at $\sqrt{s}=630$ and $1800 \mathrm{GeV}$ [inj]. All measurements are in good agreement with currently available theoretical predictions in most of the measured kinematic range.

\section{The $W$ boson Differential Production Cross Section}

Measurement of the differential cross section for $W$ boson production provides an important test of our understanding of quantum chromodynamics (QCD). Its implications range from impact on the precision determination of the $W$ boson mass to background estimates for new physics phenomena.

When the transverse momentum $\left(p_{T}^{W}\right)$ and the invariant mass $\left(M_{W}\right)$ of the $W$ boson are of the same order, the production rate can be calculated perturbatively order by order in the strong coupling constant $\alpha_{s}$. For $p_{T}^{W} \ll M_{W}$, the calculation is dominated by large logarithms $\approx \alpha_{s} \ln \left(M_{W} / p_{T}^{W}\right)^{2}$, which are related to the presence of soft and collinear gluon radiation. Therefore, at sufficiently small $p_{T}^{W}$, fixed-order perturbation theory breaks down and the logarithms must be resummed. The resummation can be carried out in transverse momentum $\left(p_{T}\right)$ space or in impact parameter $(b)$ space via a Fourier transform. Differences between the two formalisms are discussed in Ref. [i6].

Although resummation extends the perturbative calculation to lower values of $p_{T}^{W}$, a more fundamental barrier is encountered when $p_{T}^{W}$ approaches $\Lambda_{\mathrm{QCD}}$, the scale characterizing QCD processes. The strong coupling constant $\alpha_{s}$ becomes large and the perturbative

${ }^{*}$ Speaker.

${ }^{\dagger}$ For the D $\varnothing$ Collaboration 
calculation is no longer reliable. The problem is circumvented by using a cutoff value and by introducing an additional function that parameterizes the non-perturbative effects

The inclusive differential cross section for $W$ boson production is measured in the electron channel as a function of transverse momentum. We use $85 \mathrm{pb}^{-1}$ of data recorded with the $\mathrm{D} \varnothing$ detector during the 1994-1995 run of the Fermilab Tevatron $p \bar{p}$ collider.

The $W \rightarrow e \nu$ event sample is corrected for kinematic and geometric acceptance, and detector resolution. The final results for $d \sigma(W \rightarrow e \nu) / d p_{T}^{W}$ are plotted in Fig. 1, where the data are compared to the combined QCD perturbative and resummed calculation in $b$-space, computed with published values of the non-perturbative parameters $[\underline{\overline{1}} \overline{1}]$. The error bars on the data points correspond to their statistical uncertainties. The fractional systematic uncertainty is shown as a band in the lower portion of the plot, and accounts for the errors in the hadronic energy scale and resolution, the selection efficiency, and the background. An additional normalization uncertainty of $\pm 4.4 \%$ from the integrated luminosity is not included in any of the plots nor in the table.

Figure 2 shows a comparison of the differential cross section for $W$ boson production to the fixed-order perturbative calculation and to three different resummation calculations in the low $p_{T}^{W}$ region. The parameterizations of the non-perturbative region are from

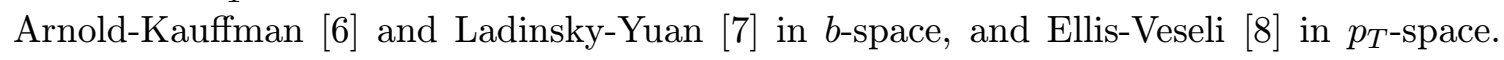
The disagreement between the data and the fixed-order prediction at low values of $p_{T}^{W}$ confirms the presence of contributions from soft gluon emission, which are accounted for in the resummation formalisms.

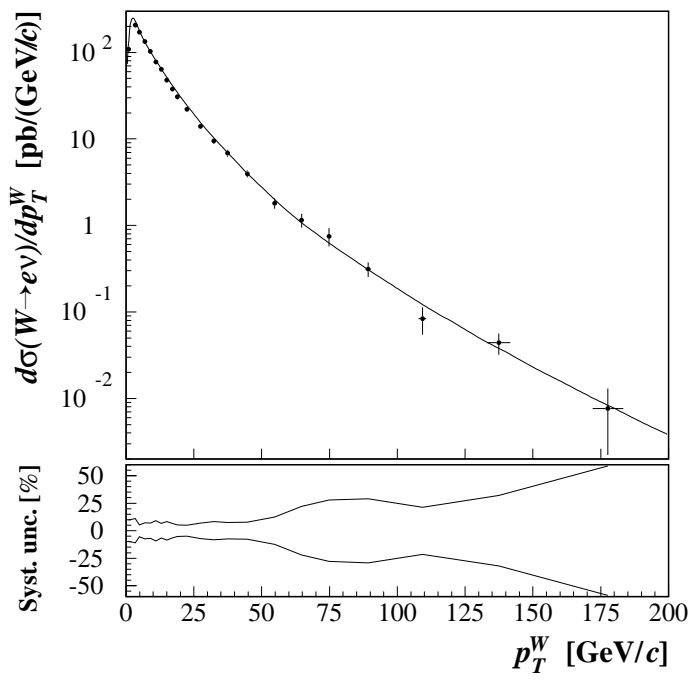

Figure 1: Differential cross section for $W \rightarrow$ ev production.

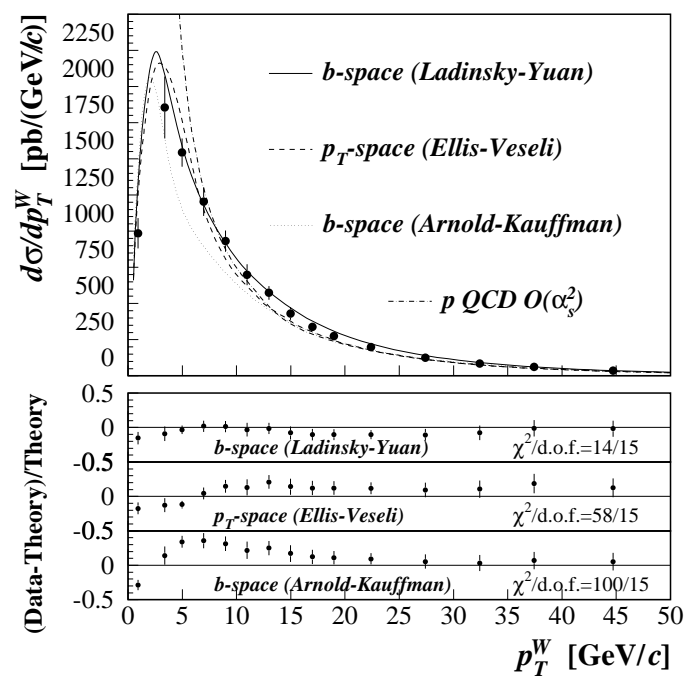

Figure 2: Differential cross section for $W$ boson production.

\section{2. $W$ and $Z p_{T}$ Ratio Measurement}

For the analyses of data taken during 1992-1996 (Fermilab Tevatron Run 1), we have used the resummed calculation of Ref. [i, $]$ itted to our observed $Z \rightarrow e^{+} e^{-}$differential 
cross section to extract the non-perturbative phenomenological parameters of the theory. The resummed calculation was then used to predict $W$ boson observables such as the electron and neutrino transverse momenta and as input to a Monte Carlo model of $W$ boson production and decay, which we used to extract the mass and production cross section of the $W$ boson.

Ref. [īi in proposes an alternative method of predicting $W$ boson observables from measured $Z$ boson quantities. This is based on the theoretical ratio of the $W$ to $Z$ boson differential cross sections with respect to variables that have been scaled by their corresponding vector boson masses. Because production properties of $W$ and $Z$ bosons are very similar, the large radiative corrections that affect the individual distributions cancel in the ratio. The ratio can therefore be calculated reliably using perturbative QCD (pQCD), with no need for resummation.

Although the alternative method reduces both the theoretical and experimental systematic uncertainties, it introduces a statistical contribution to the uncertainty from the number of events in the $Z$ boson candidate sample. Hence, once large samples of $Z$ boson events become available, it is expected that using the pQCD prediction and the wellmeasured $p_{T}^{Z}$ distribution to predict the $p_{T}^{W}$ distribution should lead to smaller overall uncertainties on the measured mass and width of the $W$ boson, relative to current methods used at hadron colliders.

The ratio of differential cross sections for the scaled $W$ and $Z$ boson transverse momenta $\left(p_{T}^{W} / M_{W}\right.$ and $\left.p_{T}^{Z} / M_{Z}\right)$ is defined as

$$
R_{p_{T}}=\left[\frac{d \sigma^{W}}{d\left(p_{T}^{W} / M_{W}\right)}\right] /\left[\frac{d \sigma^{Z}}{d\left(p_{T}^{Z} / M_{Z}\right)}\right]
$$

where $d \sigma^{V} / d p_{T}^{V}$ is the standard differential cross section for vector boson production $\sigma(p \bar{p} \rightarrow V+X)$ as a function of transverse momentum $p_{T}^{V}$. Equation $\overline{2}_{2}^{\prime} \overline{1}^{\prime}$ can be used to predict the differential cross section for $W$ bosons with respect to the non-scaled transverse momentum:

$$
\left.\frac{d \sigma^{W}}{d p_{T}^{W}}\right|_{\text {predicted }}=\frac{M_{Z}}{M_{W}} \times R_{p_{T}} \times\left.\frac{d \sigma^{Z}}{d p_{T}^{Z}}\right|_{\text {measured }} ^{p_{T}^{Z}=\frac{M_{Z}}{M_{W}} p_{T}^{W}},
$$

where $R_{p_{T}}$ is calculated using pQCD. The measurement of $R_{p_{T}}$ is compared to the cal-

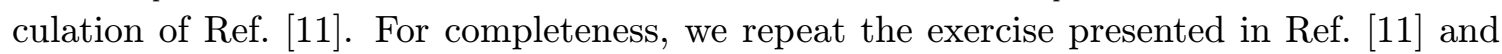

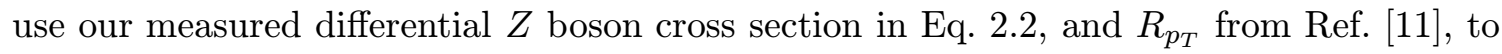
obtain the differential $W$ boson cross section and compare it to our published result [i] $\left.{ }_{1}^{1}\right]$.

From the measured $W$ and $Z$ boson differential cross sections, we extract the ratio of scaled cross sections as a function of $p_{T}, R_{p_{T}}$. The result is shown in Fig. 3. We observe that the measured $R_{p_{T}}$ agrees with the pQCD prediction [i] $\left.{ }_{1}^{1}\right]$ : the $\chi^{2}$ for the comparison between data and theory is 18.3 for 21 degrees of freedom ( $63 \%$ probability).

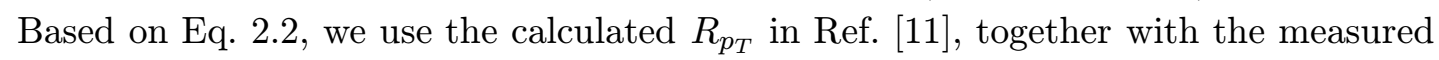
$d \sigma^{Z} / d p_{T}^{Z}$, to predict the $W$ boson transverse momentum spectrum, and compare it with our previously measured $d \sigma^{W} / d p_{T}^{W}\left[i_{1}^{i}\right]$. Fig. 4 shows the measured differential cross section 
plotted at the center of the bin. The upper and lower $68 \%$ confidence level limits for the prediction are plotted as histograms. The extracted transverse momentum distribution agrees well with the measurement.

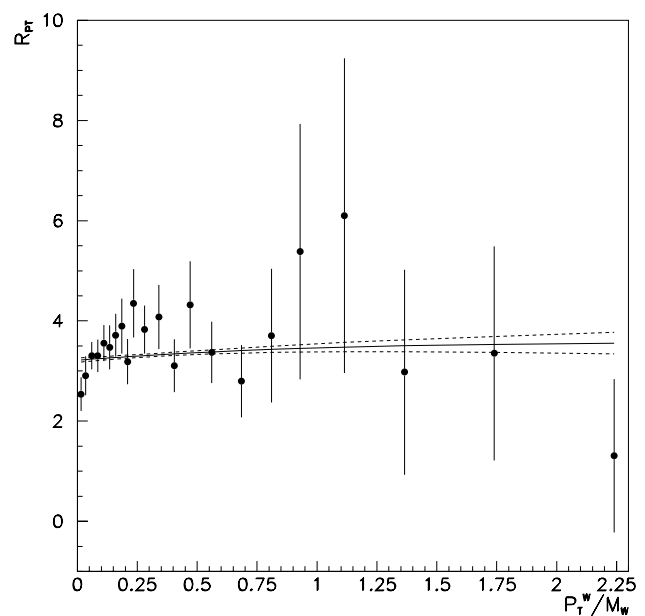

Figure 3: Ratio of scaled differential cross sections $R_{p_{T}}$ for $W$ and $Z$ production.
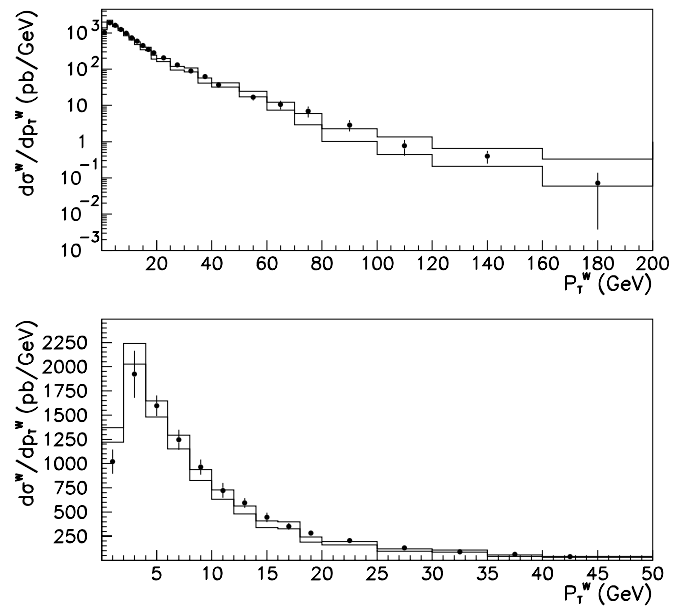

Figure 4: Differential cross section for $W$ boson production as a function of $p_{T}^{W}$.

\section{The Ratio of Direct Photon Cross Sections at $\sqrt{s}=630$ and $1800 \mathrm{GeV}$}

Within the framework of Quantum Chromodynamics (QCD), isolated single photons are direct photons: produced from the primary parton-parton interactions. A measurement of the final state photons provides a probe of QCD without additional complications from fragmentation and jet identification, providing a powerful and effective means for studying the constituents of hadronic matter.

Previous experiments, at center-of-mass energies of both $630 \mathrm{GeV}$ [1] $\overline{3} \overline{1}$ and $1800 \mathrm{GeV}$

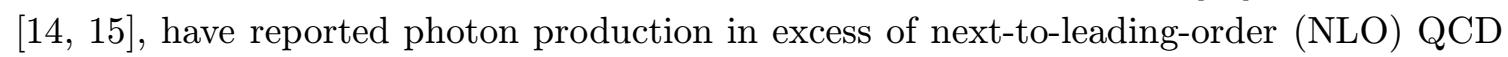
predictions at low transverse energies $\left(E_{T}^{\gamma} \lesssim 30 \mathrm{GeV}\right)$. This disagreement with data could result from gluon radiation not included in NLO calculations or because the parton distributions are not well known.

We present a new measurement of the isolated photon cross section for photons in two pseudorapidity regions, $|\eta|<0.9$ (central) and $1.6<|\eta|<2.5$ (forward), at a centerof-mass energy of $630 \mathrm{GeV}$, based on a sample of $520 \mathrm{nb}^{-1}$ recorded by D $\varnothing$ in 1995 . A ratio of the cross sections at different center-of-mass energies, 630 and $1800 \mathrm{GeV}$, is also presented. The systematic uncertainty and the sensitivity to the choice of parton distribution functions (PDF) are significantly reduced in the ratio. Photon candidates are required to have calorimeter shower characteristics consistent with that of a single electron, and must not have a track match in the drift chamber.

The fully corrected cross sections, $d^{2} \sigma / d E_{T}^{\gamma} d \eta$, are shown in Fig. 5 where they are compared with NLO QCD calculations using CTEQ5M parton distributions [i] $\left.{ }_{1}^{1} \overline{\sigma_{1}}\right]$. The error bars show all uncorrelated uncertainties, which include the statistical uncertainty, 
and uncertainties from selection criteria, and photon purity. The correlated uncertainty consists of the errors in luminosity, efficiencies, energy calibration, and acceptance. A $\chi^{2}$ test using the full covariance matrix for the experimental uncertainties gives a $12 \%(71 \%)$ probability that the theory is consistent with the data in the CC (EC) regions. Deviations between theory and data are largest at low $E_{T}^{\gamma}$ in the central region.

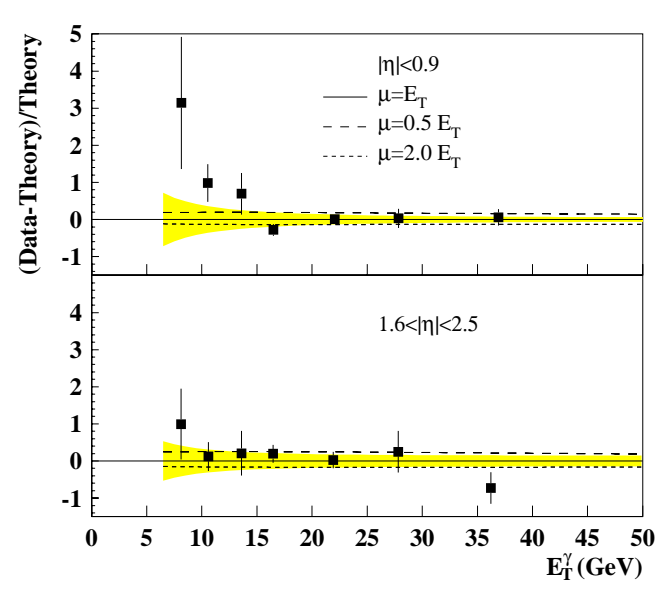

Figure 5: Comparison of the measured cross section for production of isolated photons at $\sqrt{s}=630 \mathrm{GeV}$ with NLO QCD using CTEQ5M parton distribution functions.

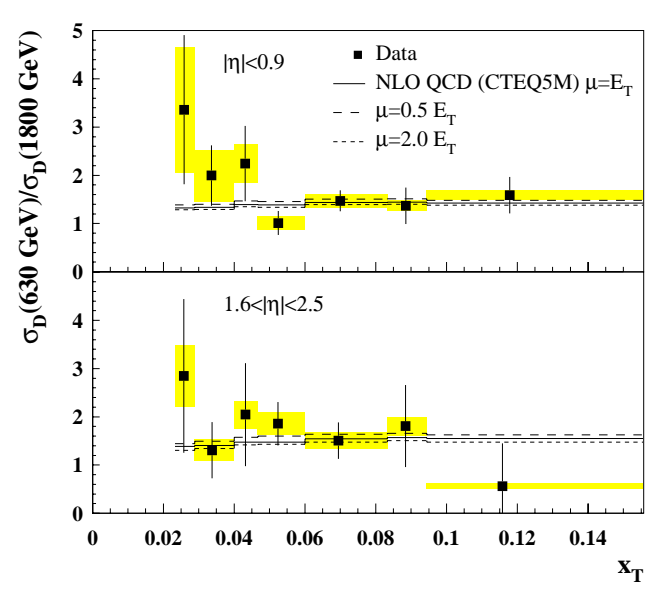

Figure 6: The ratio of the dimensionless cross sections, $\sigma_{D}(\sqrt{s}=630 \mathrm{GeV}) / \sigma_{D}(\sqrt{s}=$ $1800 \mathrm{GeV})$.

In the simple parton model, the dimensionless cross section $E_{T}^{4} \cdot E \frac{d^{3} \sigma}{d p^{3}}$, as a function of $x_{T}=\frac{2 E_{T}}{\sqrt{s}}$, is independent of $\sqrt{s}$. Although deviations from such naive scaling are expected, the dimensionless framework provides a useful context for comparison with QCD. The experimental dimensionless cross section, averaged over azimuth, becomes $\sigma_{D}=\frac{E_{T}^{3}}{2 \pi} \cdot d^{2} \sigma / d E_{T} d \eta$. The ratio $\sigma_{D}(\sqrt{s}=630 \mathrm{GeV}) / \sigma_{D}(\sqrt{s}=1800 \mathrm{GeV})$ is determined by combining the cross section reported here with the $\mathrm{D} \varnothing$ measurement at $\sqrt{s}=1800$ $\mathrm{GeV}$ [1] prediction.

The probability of agreement from a $\chi^{2}$ test between data and theory is $49 \%$ (89\%) in the CC (EC) region. The deviations at low $x_{T}$ are not significant in light of our combined statistical and systematic uncertainties, and there exists good agreement between the measured ratio and theory.

\section{Summary and Prospects}

The new $\mathrm{D} \varnothing$ gauge boson results are in good agreement with theoretical predictions. The deviations at low $p_{T}$ are not significant. The study of the $W, Z, \gamma$ production mechanisms is important as a direct test of the standard model, to improve the understanding of the background in top quark and Higgs boson production, as well as to control the systematics in precision measurements such as the $W$ mass. Run 2 has started and we expect $2 \mathrm{fb}^{-} 1$ 
of data by year 2003. The much larger number of gauge bosons will allow us to reduce systematics in both the measurements and the predictions. We will also be able to reduce uncertainties in the direct photon cross sections (dominated by the photon purity of the sample), as well as to extend the kinematic range of the measurement.

\section{References}

[1] V. M. Abazov et al.., (DØ Collaboration), Phys. Lett. B 513, 292 (2001).

[2] B. Abbott et al., (DØ Collaboration), Phys. Rev. Lett. 80, 5498 (1998).

[3] B. Abbott et al.., (DØ Collaboration), Phys. Rev. D 61, 032004 (2000), and Phys. Rev. Lett. 84, $2792(2000)$.

[4] V. M. Abazov et al.., (DØ Collaboration), Phys. Lett. B 517, 299 (2001).

[5] V. M. Abazov et al.., (DØ Collaboration), hep-ex/0106026, accepted by Phys. Rev. Lett., (2001).

[6] P. B. Arnold and R. P. Kauffman, Nucl. Phys. B349, 381 (1991).

[7] G. A. Ladinsky and C. P. Yuan, Phys. Rev. D 50, 4239 (1994).

[8] R. K. Ellis and S. Veseli, Nucl. Phys. B511, 649 (1998).

[9] L. Lindemann and G. Zech, Nucl. Instrum. Methods A 354, 516 (1995).

[10] G. D. Lafferty and T. R. Wyatt, Nucl. Instrum. Methods A 355, 541 (1995).

[11] W. T. Giele and S. Keller, Phys. Rev. D 57, 4433 (1998).

[12] T. Affolder et al.., (CDF Collaboration), Phys. Rev. Lett. 84, 845 (2000)

[13] J. Alitti et al. (UA2 Collaboration), Phys. Lett. B 263, 544 (1991); R. Ansari et al. (UA2 Collaboration), Z. Phys. C 41, 395 (1988).

[14] B. Abbott et al. (DØ Collaboration) Phys. Rev. Lett. 84, 2786 (2000).

[15] F. Abe et al. (CDF Collaboration), Phys. Rev. Lett. 73, 2662 (1994); F. Abe et al. (CDF Collaboration), Phys. Rev. D 48, 2998 (1993).

[16] H. Baer, J. Ohnemus, and J.F. Owens, Phys. Rev. D 42, 61 (1990); W. Vogelsang and A. Vogt, Nucl. Phys. B 453, 334 (1995).

[17] CTEQ5M, CTEQ5HJ, MRST, MRSTg $\uparrow$, and MRSTg $\downarrow$ were compared. For MRST, see A.D. Martin et al. Eur. Phys. J. C 14, 133 (2000). 